\title{
ON HILBERT'S INEQUALITIES WITH ALTERNATING SIGNS
}

\section{CHANG-JiAn ZHAO AND WING-SUM CHEUNG}

Abstract. Some new Hilbert type inequalities with alternating signs are established. These also generalize some existing results of Hilbert type inequalities in the literature.

Mathematics subject classification (2010): 26D15.

Keywords and phrases: Hilbert's inequality, Hilbert's inequality with alternating signs, Hölder's inequality, Jensen's inequality, Young's inequality.

\section{REFERENCES}

[1] G. H. Hardy, J. E. Littlewood and G. Pólya, Inequalities, Cambridge Univ. Press, Cambridge, 1934.

[2] D. S. Mitrinović, Analytic Inequalities, Springer-Verlag, Berlin, New York, 1970.

[3] B. C. YANG, On Hilbert's integral inequality, J. Math. Anal. Appl. 220 (1988), 778-785.

[4] M. Z. GAO, T. LI, Some improvements on Hilbert's integral inequality, J. Math. Anal. Appl. 229 (1999), 682-689.

[5] M. Z. GAO, B. C. YAng, On the extended Hilbert's inequality, Proc. Amer. Math. Soc. 126 (1998), $751-759$.

[6] J. C. KuAnG, On new extensions of Hilbert's integral inequality, J. Math. Anal. Appl. 235 (1999), 608-614.

[7] J. C. Kuang, L. Debnath, On Hilbert type inequalities with non-conjugate parameters, Appl. Math. Lett. 22 (2009), 813-818.

[8] M. Krnić, J. PeČArić, Extension of Hilbert's inequality, J. Math. Anal. Appl. 324 (2006), 150-160.

[9] Z. Lv, M. Z. Gao, L. Debnath, On new generalizations of the Hilbert integral inequality, J. Math. Anal. Appl. 326 (2007), 1452-1457.

[10] B. G. PAChPATte, Inequalities similar to certain extensions of Hilbert's inequality, J. Math. Anal. Appl. 243 (2000), 217-227.

[11] G. A. Anastassiou, Hilbert-Pachpatte type fractional integral inequalities, Math. Compu. Mode. 49 (2009), 1539-1550.

[12] J. Jin, L. Debnath, On a Hilbert-type linear series operator and its applications, J. Math. Anal. Appl. 371 (2010), 691-704.

[13] B. C. YANG, A half-discrete Hilbert-type inequality with a non-homogeneous kernel and two variables, Mediterranean J. Math. 10 (2) (2013), 677-692.

[14] G. D. Handley, J. J. Koliha AND J. E. PeČARIĆ, New Hilbert-Pachpatte type integral inequalities, J. Math. Anal. Appl. 257 (2001), 238-250.

[15] Z. T. XIE, A new reverse Hilbert-type inequality with a best constant factor, J. Math. Anal. Appl. 343 (2008), 1154-1160.

[16] C. J. Zhao, L. Debnath, Some new inverse type Hilbert integral inequalities, J. Math. Anal. Appl. 262 (2001), 411-418.

[17] C. J. ZhaO, W. S. Cheung, Reverse Hilbert's type integral inequalities, Math. Inequal. Appl. 17 (4) (2014), 1551-1561.

[18] B. G. PAChPATte, On some new inequalities similar to Hilbert's inequality, J. Math. Anal. Appl. 226 (1998), 166-179.

[19] G. S. Davies, G. M. Peterson, On an inequality of Hardy's (II), Quart. J. Math. 15 (1964), 35-40. 